\title{
MUERTES POR LESIONES PRODUCIDAS POR ARMAS DE FUEGO Y EXPLOSIVOS, REGISTRADAS EN EL INSTITUTO DE CIENCIAS FORENSES Y CRIMINALÍSTICA DE QUITO DESDE EL AÑO 2017 AL 2019
}

\author{
Román María del Cisne, López Bravo Marcela², Mejía Suarez Eliana ${ }^{3}$, Ordoñez Mullo Luis ${ }^{4}$. \\ cisne-1991@hotmail.com¹, marcelalopeszbravo@outlook.com², elianam-17@hotmail.com³, \\ luisgustavo678@gmail.com ${ }^{3}, \operatorname{login}^{2}$ \\ https://orcid.org/0000-0002-1045-40011 , https://orcid.org/0000-0002-0777-76942, \\ https://orcid.org/0000-0003-3848-46483, https://orcid.org/0000-0003-4629-98054 \\ Hospital General IESS Machala, Hospital Básico de Alamor \\ Machala-Ecuador
}

Recibido (13/07/20), Aceptado (28/07/20)

\begin{abstract}
Resumen: Las muertes violentas en Ecuador representan una problemática social importante, en este trabajo hemos aplicado una metodología de estudio observacional, retrospectiva, transversal, no experimental, la población fue conformada por el total de personas fallecidas que fueron registradas por el Instituto de ciencias forenses y criminalística, y la muestra se consideró las muertes por lesiones producidas por armas de fuego y explosivos desde enero del 2017 a diciembre del 2019, obteniendo como resultados que el $100 \%$ de muertes de esta modalidad fueron catalogadas como muertes violentas y a todos ellos se les realizó autopsia, más del $80 \%$ de las víctimas fueron de sexo masculino, el rango de edad con mayor cantidad de víctimas oscila entre los 36 y 64 años de edad, las lesiones mortales por armas de fuego fueron en su mayoría dirigidas a cavidades craneal y toraco-abdominal, mientras que por explosivos las lesiones fueron generalizadas provocando muerte instantánea.
\end{abstract}

Palabras Clave: Lesiones, muertes, armas de fuego, explosivos.

\section{DEATHS FROM INJURIES CAUSED BY FIREARMS AND EXPLOSIVES, REGISTERED AT QUITO INSTITUTE OF FORENSIC SCIENCES AND CRIMINALISTICS FROM 2017 TO 2019}

\begin{abstract}
Violent deaths in Ecuador represent an important social problem, in this work we have applied an observational, retrospective, cross-sectional, non-experimental study methodology, the population was made up of the total number of deceased persons who were registered by the Institute of Forensic and Criminal Science, and the sample considered deaths from injuries caused by firearms and explosives from January 2017 to December 2019, obtaining as a result that $100 \%$ of deaths in this modality were classified as violent deaths all of them underwent an autopsy, more than $80 \%$ of the victims were male, the age range with the highest number of victims ranged from 36 to 64 years of age, fatal injuries from firearms were mostly directed at cranial and thoraco-abdominal cavities, while explosives injuries were widespread causing instant death
\end{abstract}

Keywords: Injuries, deaths, firearms, explosives. 


\section{I.INTRODUCCIÓN}

La violencia armada hace referencia a un tipo de violencia generada por el uso de armas (bombas, explosivos, minas, etc). Este tipo de violencia es más mortal que otros tipos, debido a que las armas de fuego por lo general producen lesiones mortíferas y reducen al mínimo la posibilidad de poder escapar o que alguien pueda intervenir para ofrecer protección. A nivel mundial, cientos de personas mueren cada año a causa de un disparo (2380.840 victimas), incluso se estima que la mitad de todos los homicidios fueron ocasionados por un arma de fuego, [1].

Afecta en su mayoría a estados en los que se evidencian niveles altos de inequidad en el ingreso, incapacidad para satisfacer necesidades básicas, debilidad institucional y sistemas judiciales precarios. Este tipo de violencia ocurre en escenarios lejanos a los de una guerra, pero cercanos al crimen organizado y al delito. En el año 2017, los países del continente americano (incluye Estados Unidos y Canadá), registraron el $37 \%$ del total de homicidios intencionales ocurridos en el mundo (173.000), aunque solo suman el 13\% de la población mundial. En otras palabras siendo América el tercer continente más poblado del planeta (después de Asia y África), tiene el mayor número de homicidios a escala global, y al menos las dos terceras partes de esta violencia se cometió con armas de fuego. Estas cifras demuestran que el continente americano, además de ser el más violento del mundo, es el lugar en el cual las armas de fuego son utilizadas para matar a la mayor cantidad de gente [2].

En los últimos años en Ecuador ha habido un descenso de las muertes por arma de fuego. Sin embargo a pesar de ello sigue siendo un problema latente en la sociedad. Los homicidios incluyen también los delitos de asesinato, femicidio y sicariato.

Según las cifras nacionales de 2018, el 72,51\% de estas muertes han sido asesinatos; el 20,54\%, homicidios; el 6,65\%, femicidios; y el 0,30 de los casos han sido sicariato. Por arma, el $45,47 \%$ de los homicidios se efectuaron con arma de fuego, el $36,40 \%$ con arma blanca, el 0,15\% por sustancias, entre otros. Por edades, el grupo con más víctimas tiene entre 20 y 29 años. Alcanzan el 35\% de los casos. Luego está el grupo entre 30 y 39 años $(25,53 \%)$; los de $40-49$ años $(13,89 \%)$, 15-19 años (5,59\%), entre otros. [4].

Por todo lo anterior descrito la finalidad del presente estudio es identificar el porcentaje de muertes producidas por lesiones por armas de fuego y explosivos en la ciudad de Quito durante el periodo comprendido entre los años 2017 al 2019 para poder analizar las variables, cuyos datos serán tomados de las estadísticas del centro de Ciencias Forenses y Criminalística de la Ciudad de Quito.

\section{II.DESARROLLO}

\section{A.Lesiones por arma de Fuego.}

Las lesiones por armas de fuego son un conjunto de alteraciones que se producen en el organismo como efecto de los elementos que integran un disparo. En estados unidos cada año fallecen de 30.000 a 50.000 personas de manera secundaria a heridas por proyectil de arma de fuego y constituyen la primera causa de muerte en las primeras 2 décadas de la vida. Es considerada como un problema de salud pública, una grave epidemia social, que se manifiesta no solo en número de fallecidos o las tasas de mortalidad, sino también en la cantidad, intensidad y variedad de formas en que se ejerce, así como en el deterioro de la calidad de vida y en las condiciones de salud de la población [8].

\section{B.Tipos de muertes violentas.}

Se han establecido 3 tipos de muertes violentas que pueden ser por: homicidio, suicidio y accidente; así como los factores asociados a cada una de las tipologías para lograr determinar en la investigación de la escena ante cuál de ellas nos encontramos.

Las lesiones causadas por proyectiles de arma de fuego, tienen una gran variabilidad por su mecanismo de producción, sitio donde se originó la lesión, regiones anatómicas afectadas, el grado de lesión y la evolución que puedan tener los órganos comprometidos [7].

\section{C.Orificio de entrada.}

Se define como la lesión que se produce en la piel por penetración de un proyectil más los elementos concurrentes y formados por efecto del disparo.

-Características anatomopatológicas:

Constantes: Orificio de penetración, anillo erosivo o de contusión, anillo de enjugamiento [10].

-Secundarias: Tatuaje, zona de ahumamiento.

La localización del orificio de penetración puede ser típica en área corporal evidente, atípica en áreas como boca, oído, angulo interno del ojo. En cuanto al tamaño si es menor que el diámetro del proyectil es lo común, mayor que el diámetro del proyectil (cuando este llega deformado en disparos de corta distancia), mecanismo: a) ensuciamiento del extremo anterior del proyectil al pasar por el cañón del arma b) enjugamiento de esta suciedad al pasar por el orifico de entrada de la herida, caracteres: a) la localización sobre la piel o la ropa, 
b) forma circular (disparo perpendicular) o semulunar (disparo oblícuo)

Importancia médico legal.- Diagnóstico del orifico de entrada, determinación de la naturaleza de la herida, determinación de la dirección del disparo

Collarete erosivo (cintilla de contusión) fue descrito por Piedelievre como signo característico del orificio de entrada a cualquier distancia del disparo, siendo el resultado de la excoriación epidérmica alrededor del orificio de penetración. Además, el diámetro del collarete erosivo no indica el calibre de la bala.

Collarete de limpieza (anillo de enjugamiento) está caracterizado por un ribete negro, circular, situado en el borde del orifico de entrada, junto al collarete erosivo, por roce de la superficie de la bala cuya extremidad anterior transporta las escorias metálicas, la herrumbre y las suciedades recogidas al pasar a lo largo del cañón. La cintilla de contusión más el collarete de limpieza forman el anillo de Fisch.

Tatuajes es identificado como la zona de puntos oscuros en la piel alrededor del orificio de entrada, se constituye por los fragmentos de pólvora no deflagrada que se incrustan la piel, que no van a desaparecer con el lavado.

Zona de ahumamiento (falso tatuaje) consiste en una aureolo nebulosa que representa el deposito del humo producto de la combustión.

\section{D.Tipos de orificio de entrada}

Disparos de contacto, disparos de corta distancia y disparos de larga distancia, en el orificio de entrada en disparo de contacto es aquel en el cual el disparo se realiza directamente sobre la piel pueden apreciarse signos en la ropa y en la piel. Pueden denominarse disparo a boca de jarro o disparo a quemarropa, los signos sobre el cuerpo son: signo de Puppe- Werkgrtner: se identifica el contorno de boca de fuego sobre la piel, signo de boca de mina de Hofmann: se aprecian bordes irregulares, de coloración negruzca y pueden presentar desgarros, signo de Benassi: se evidencia material de coloración negra sobre la superficie ósea.

Los signos que pueden apreciarse en ropas son: signos de calcado: la piel imita las características de la ropa sobre la cual se dispara, signo de deshilachamiento crucial: se evidencia prendas desgarradas en forma de cruz con borde de coloración negruzca, signo de escarapela: formación de dos anillos concéntricos formados por humo, circundantes a la perforación del orificio producida en la ropa, separados por un halo claro, en el orificio de entrada en disparo de corta distancia la boca de fuego del arma no se encuentra muy distante de la piel, la distancia máxima va a depender del tipo de arma utilizada por lo general es menor de tres yardas o 2.7 metros aproximadamente, en el orificio de entrada en disparo de larga distancia se produce cuando el proyectil produce lesiones a más de tres yardas de distancia, se caracteriza por presentar múltiples heridas, diseminadas, con recorridos cortos, y ausencia de orificios de salida, posterior a pasar el primer metro las esferas se dispersan (llamada rosa de dispersión) y cada perdigón puede actuar como un proyectil único e independiente produciendo características propias del orificio de entrada.

\section{E.Trayecto}

El trayecto del proyectil dentro del cuerpo desde que produce el orificio de entrada hasta que atraviesa el orifico de salida en el caso que se presentara. Esto nos ayuda a identificar órganos que pudieran verse afectados y lugares donde se puede habar alojado la bala, el recorrido suele ser una línea recta, pero puede desviarse o migrar si entra en contacto con hueso o algún órgano solido pudiendo presentar cambios de dirección inesperados.

\section{F.Orificio de Salida.}

Es la lesión provocada por el proyectil posterior a travesar el cuerpo, puede estar presente o no, y tiene características propias, puede o no estar presente, forma irregular (estrellada), puede presentar desgarramiento de tejido, pero también puede presentar forma circular, ovalada, o hendidura lineal, bordes evertidos, hernia de grasa subcutánea, ausencia de collarete, no presenta tatuaje ni ahumamiento, ni anillo de Fisch.

\section{G.Heridas por explosiones.}

Las lesiones por explosivos han sido clasificadas teniendo en cuenta orientaciones clínicas para atención de victimas sobrevientes, como las planetadas por el National Center for Injury Prevention and Control- NCIPC (Centro Nacional de Prevencion y Control de Lesiones) [11] [12].

El mecanismo general de producción de las lesiones durante una explosión, por explosivos de alto o bajo poder, es la conversión de un sólido o líquido a gas, la rápida expansión de este gas con el aumento de la presión y formación de una onda explosiva que atraviesa los cuerpos y objetos y de una onda de viento que desplaza los mismos. [11].

El término blast engloba las lesiones anatómicas y el síndrome clínico provocado por la exposición del organismo a los efectos de una onda de choque ocasionada por una explosión, consta esquemáticamente de tres componentes: la onda de choque, vientos expansivos y 
el calor.

Las lesiones Causadas por la explosión se divide en cuatro categorías relacionadas con la onda de choque que son: primarias, secundarias, terciarias y cuaternarias e incluso quinquenarias.

En los incidentes con víctimas en masas que implican explosiones, se identifican 3 zonas concéntricas: Epicentro de onda expansiva, perímetro secundario, periferia de onda expansiva. [13]

Se toma en cuenta un nuevo sistema de clasificación que permite la agrupación de lesiones anatómico- patológicas de manera sistemática y documentada, útil a los médicos que requieran valoraciones y diagnósticos en patología forense. A continuación definiremos las lesiones por onda explosiva según la Clasificación de Bogotá. [11]

Lesiones primarias por onda explosiva o blast, son lesiones producidas mediante el paso de la onda por el cuerpo y la inmediata destrucción, asociadas o no a la interfase aire- liquido/solido, las mismas que pueden ser lesiones generalizadas y descritas como desintegración corporal y daño corporal sin desintegración, son lesiones que producen gran destrucción corporal y muerte instantánea.

Lesiones de órganos con interfase aire/liquido o sólido/líquido: pulmón, oído, tracto, gastrointestinal, fracturas de cara relacionadas con los senos paranasales y la ruptura de cavidades cardiacas, amputaciones y fracturas de extremidades producida por la onda relacionadas con la cercanía al artefacto explosivo, quemaduras por viento de calor y/o radiación, denominadas comúnmente como quemaduras por flash.

Lesiones secundarias son producidas por misiles secundarios propulsados por la onda explosiva y pueden desprenderse de la misma bomba o del medio y consisten en heridas generalmente pequeñas, excoriaciones, hematomas y laceraciones de tejidos que se relacionan con fragmentos encontrados dentro del cadáver.

Lesiones terciarias, estas lesiones están relacionadas con el lanzamiento corporal que incluye los traumas por aceleración - desaceleración y/o por aplastamiento secundario por colapso de edificaciones. Consisten en traumas contundentes, generalmente severos

Lesiones cuaternarias se producen por eventos desencadenados por la explosión luego que ha pasado la onda explosiva y ya ha destruido cuerpos y objetos.

Para la realización de la valoración médico legal de lesiones producidas por armas de fuego y explosivos se debe seguir la misma sistematización que para las muertes violentas.

Estudio del lugar de los hechos y levantamiento del cadáver, Cuando ya se ha terminado de la inspección ocular, documentación fotografía y recogida de indicios y la zona a sido acordonada y no hay peligro de nuevas explosiones, el médico forense procede a realizar la primera inspección del cadáver, de manera general se evidencian las lesiones que presenta, si existen mutilaciones, aplastamientos, identificar los fenómenos cadavéricos para poder determinar el tiempo en que ha ocurrido la muerte. Se pueden recoger también fragmentos anatómicos, muestras para su análisis en el laboratorio de criminalística, y traslado del cadáver a la morgue para realización de la autopsia [11].

El examen externo realizado por el médico forense, este examen incluye el estudio de las ropas, elementos extraños que se encuentren impregnados en el cuerpo, se debe describir con exactitud las lesiones (quemaduras, heridas, escoriaciones, contusiones, laceraciones), se pueden usar métodos radiológicos para poder determinar otras posibles lesiones como fracturas o incrustaciones de elementos metálicos.

Examen interno se podrá realizar en dependencia de la integridad del cadáver, si el cadáver se encuentra integro se puede realizar una disección cuidadosa para localizar las lesiones más graves u objetos metálicos dentro de cavidades. En el caso de solo obtener fragmentos de la víctima se procederá al análisis de cada fragmento e intentar realizar una reconstrucción para proceder con la identificación del cadáver [11].

a toma de muestras nos ayuda a poder identificar el tipo de explosivo usado, análisis de la ropa o fragmentos incrustados para determinar su origen, análisis químico- toxicológico, para poder realizar la datación de la muerte o identificación de víctimas.

La reconstrucción del cadáver en muchos de los casos no es posible realizar la reconstrucción del cadáver, y solo es factible poder realizar la disección de restos

El informe de autopsia debe ser completo y conciso, detallando cada paso que se realiza en el estudio médico legal, llegando a una conclusión que nos oriente a la etiología y data de la muerte.

\section{III.METODOLOGÍA}

Se trata de un estudio observacional, retrospectivo, transversal, no experimental, la población fue conformada por el total de personas fallecidas que fueron registradas por el Instituto de ciencias forenses y criminalística, y la muestra se consideró las muertes por lesiones producidas por armas de fuego y explosivos desde enero del año 2017 a diciembre del 2019.

Los criterios de inclusión fueron las muertes que se encuentren en el registro del instituto de ciencias forenses y criminalística de la ciudad de Quito, hombres o mujeres cuya causa de muerte fue por armas de fuego y 
explosivos, los criterios de exclusión fueron las muertes que no se encuentren en el registro del instituto de ciencias forenses y criminalística de la ciudad de Quito.

Personas cuya causa de muerte no fue por armas de fuego, personas cuya muerte haya sido por enfermedad $\mathrm{u}$ otros tipos de muertes violentas.

\section{IV.RESULTADOS}

En los últimos tres años se evidenció que el total de las lesiones por armas de fuego y explosivos $100 \%$ fueron efectos por muertes violentas y el $0 \%$ fueron accidentales, al 100\% de los cadáveres se les realizó la respectiva valoración médico legal y autopsia. El porcentaje más alto de muertes se encontró en el sexo masculino en los 3 años por encima del $80 \%$, el rango de edad en el que se produjeron más muertes fueron de 36 a 64 años y el menor porcentaje fue en menores de 18 años representando menos del 10\% de los casos, por último se estableció la frecuencia de las áreas anatómicas donde se encontraron las lesiones en los cadáveres, ubicamos a los explosivos como el productor de lesiones en áreas anatómicas mayormente afectadas, ya que este tipo de armas provocan daño en la totalidad del cuerpo de la víctima pudiendo ocasionar desintegración o desmembramiento, por ende muerte instantánea, seguida de lesiones ubicadas en cavidades toraco-abdominal y craneal, cabe indicar que ninguna víctima mortal presento lesiones a nivel de extremidades. Por ultimo según las áreas anatómicas encontramos que el $0 \%$ de los fallecidos presentaron lesiones a nivel de extremidades, siguiendo las lesiones a nivel craneal, luego en cavidad toraco-abdominal, con mayor porcentaje las lesiones por explosivos ya que estas afectan en su totalidad a la víctima, provocando lesiones mortales instantáneas.

\section{V.CONCLUSIONES}

Con este trabajo hemos podido apreciar que las muertes violentas producidas por armas de fuego y explosivos, es un problema vigente y difícil de controlar ya que no todos los ciudadanos portan el permiso de armas respectivos y además el uso y comercialización de explosivos no está permitido, resaltamos la importancia de llevar una base de datos local y nacional, esto nos permite tener un conocimiento de la frecuencia con la que estos delitos suceden, en la literatura reporta que las lesiones y muertes por explosivos son poco frecuentes pero con estos datos nos podemos dar cuenta que las muertes por explosivos si representan un problema real, y que por su letalidad poco o nada se puede hacer para poder ayudar a la víctima. Se podría realizar una clasificación dentro de las muertes por explosivos, identificar el tipo de explosivo que se ha usado para ir determinan- do cuales son los que de usan con mayor frecuencia.

\section{REFERENCIAS}

[1]UNODC, «Global study on homicide.,» 2013.

[2]UNODC, «United Nations Office on Drugs and Crime,» World Drug Report, 2019.

[3]C. M. Á. Velasco y G. J. Pontón, «Lesiones con armas de fuego: sobrevivir a las balas en Ecuador.,» Estado \& comunes, revista de políticas y problemas públicos, vol. 1 (10), 2020.

[4]J. P. Pinto, «Más que plomo,» Perfil Criminológico, vol. 17, 2015.

[5]G. J. González Pérez, M. G. Vega López, C. E. Cabrera Pivaral, A. Vega López y A. Muñoz de la Torre, «Mortalidad por homicidios en México: tendencias, variaciones socio-geográficas y factores asociados,» Ciência \& Saúde Coletiva, vol. 17, pp. 3195-3208, 2012.

[6]J. Cavazos, J. Palacios, F. Reyna, N. Álvarez, L. Alatorres y G. Muñoz, «Epidemiología de las lesiones por proyectil de arma de fuego en el Hospital Universitario "Dr. José Eleuterio González" de la,» Cirugía y Cirujanos, vol. 85, pp. 41-48, 2017

[7]O. S. Castro, La muerte violenta. inspeccion ocular y cuerpo del delito., LA LEY., 2004.

[8] M. Zurbarán, F. Calle y R. Restrepo, «Descripción de lesiones por explosivos: revisión de la literatura, reporte de caso y propuesta de nueva clasificación.,» vol. 1(2), pp. Case reports, 1(2), 1-68., 2015.

[9]National Center for Injury Prevention and Control, «Explosions and Blast injuries: A primer for Clinicians.,» Atlanta: Centers for Disease Control and Prevention, 2006.

[10]P. Pasquier, B. Lenoir, y B. Debien, «Blast y lesiones por explosión.,» EMC-Anestesia-Reanimación,, vol. 40 (1), pp. 1-12., 2014.

[11]M. Carnicero y M. Baigorri, «Muerte por explosión: cuestiones y sistemática médico-forenses.,» Cuad. med. forense, pp. 39-52, 2002. 


\section{RESUMEN CURRICULAR}

Marcela López, Médico General, otorgado, por la Universidad Tecnológica Equinoccial, Quito-Ecuador, actualmente cursa residencia médica en el área de medicina interna, en el Hospital General IESS Machala.

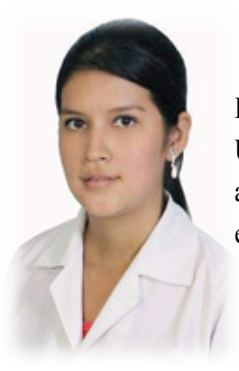

Luis Ordoñez, Médico General, otorgado por la Universidad Nacional de Loja, Loja-Ecuador, actualmente cursa residencia médica en el área de emergencia, en el Hospital Básico de Alamor.

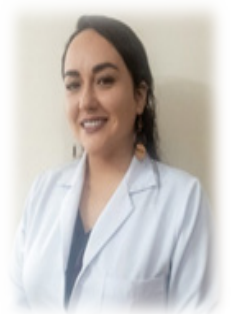

María del Cisne Román, Médico General, otorgado por la Universidad Técnica de Machala, MachalaEcuador, actualmente cursa residencia médica en el área de medicina interna, en el Hospital General IESS Machala.

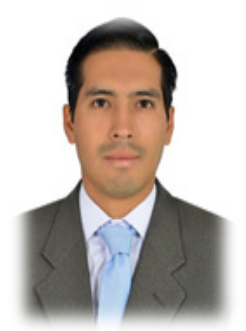

Eliana Mejía, Médico General, otorgado por la Universidad Técnica de Machala, MachalaEcuador, actualmente cursa residencia médica en el área de emergencia pediátrica, en el Hospital General IESS Machala. 